\title{
Chapter 20 \\ Effect of Titanium Surface Modifications of Dental Implants on Rapid Osseointegration
}

\author{
Ting Ma, Xiyuan Ge, Yu Zhang, and Ye Lin
}

\begin{abstract}
The initial cellular response to the dental implant is essential for the subsequent tissue regeneration around the foreign implant surface. There are many cells and proteins involved in the integration process which leads to the final osseointegration between implants and peri-implant bone tissue. With regard to materials used in dental implants, titanium is a prevalent biomaterial applied in orthopedic or dental implants due to its premium mechanical and biological properties and osteoconductivity. The roughness and chemical composition of the titanium surface affect the process and rate of the osseointegration of dental implants. Different studies on the effect of roughness and wettability of titanium surface on the process of early events in the osseointegration are reviewed in this article. In addition, in order to accelerate this wound-healing process, varied surface topography and chemical composition have been produced depending on different types of surface modifications. The desirable dental implant surface design caters for the development of implantology for immediate loading and the improvement of long-term stability. An appropriate understanding of the interaction between cells and implant surfaces is essential for the future design of new surface which could enhance the speed and stability of osseointegration of dental implants.
\end{abstract}

Keywords Osseointegration $\bullet$ Surface modification $\bullet$ Bone-implant interface

T. Ma $\bullet$ Y. Zhang $(\bowtie) \bullet Y$. Lin

Department of Oral Implantology, Peking University, School and Hospital of Stomatology, 22th South Avenue, Zhongguancun, Haidian District, Beijing 100081, People's Republic of China e-mail: zhang76yu@163.com

X. Ge

Central laboratory, Peking University, School and Hospital of Stomatology,

22th South Avenue, Zhongguancun, Haidian District, Beijing, People's Republic of China 


\subsection{Introduction}

In the 1960s, Per Ingvar Brånemark and his colleagues accidentally discovered that titanium implants and peri-implant bone tissues could be firmly bonded and there was no fibrous tissue between the interface according to intravital microscopy observation of bone defects around the microcirculation in the experiment, and they named this phenomenon "osseointegration." After continuous revision, in 1985, professor Brånemark redefined "osseointegration" as a direct interface between living bones and the implant surface, and this functional connection can be loaded $[1,2]$. Due to its premium mechanical properties, chemical stability and biocompatibility, titanium and its alloys have been widely used in dental and orthopedic implants [3]. When titanium and its alloys are exposed in the air within a very short time, a dense oxide layer will be formed on its surface. This oxide layer does not only boost good biocompatibility but also makes this metallic material biologically inert, and the implant surface treatment is usually referred to the modification on this oxide layer [4]. In recent years, with technological development of implantology, techniques that shortened treatment time and lower failure risk are urgently demanded. In this regard, the osseointegration is affected by the surface characteristics of the titanium implant, such as roughness, wettability, the chemical composition, and so on $[5,6]$. Therefore, the osseointegration biological process, the interaction between cells and implant surface, and some commonly used dental implant surface modification methods are reviewed in this article.

\subsection{Cellular Events at the Bone-Implant Interface}

During the biological process of osseointegration, a great number of different types of cells involved in this process exert an important role. Bone-implant integration is similar to the wound-healing process, which can be divided into four distinct phases: hemostasis phase, inflammatory phase, proliferative phase, and remodeling phase. Once the implant was placed into the prepared site, hemostasis began. Subsequently, growth factors and matrix proteins were activated and released from the injured tissue during the drilling process. Within a few seconds to hours, there was a water layer on the implant surface and extracellular matrix proteins were adsorbed onto the surface. Among these proteins, some of them such as vitronectin and fibronectin have interactions with the following host inflammatory response. Moreover, aggregated fibrinogen matrix facilitates more platelet adhesion and aggregation, and then the release of vasoactive substances and chemokines from the platelets symbolizes the beginning of inflammatory response [7-10] (Fig. 20.1).

The initial stage of the inflammatory phase is activated by the innate immune system. Once adhering to the surface of the implant, neutrophils secrete proinflammatory cytokines, collagenase, and other enzymes to remove the foreign bacteria. If the bacteria have not been cleared or a large number of bacteria still exist, 


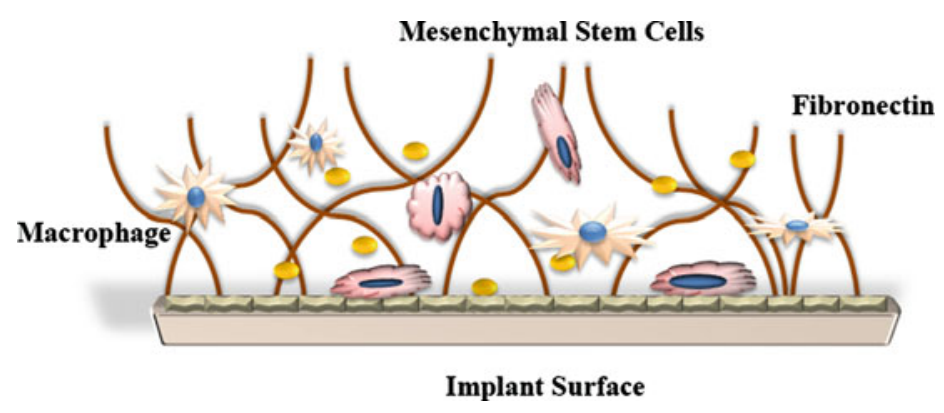

Fig. 20.1 Schematic showing fibrin clot formation on the implant surface and macrophages and MSCs play important roles in this process

the duration of cellular innate immune response will be extended, which would damage the surrounding normal tissue. Therefore, prolonged cellular innate immune response would also impede the wound healing. To sum up, immune and inflammatory responses play an important role during the wound-healing process of foreign implants [9]. In addition, another cell that is adsorbed onto the implant surface is the macrophage. Macrophages play an important role in phagocytosis of necrotic tissue, secretion of fibroblast growth factor, and angiogenic growth factors [11]. As osteoblast precursor cells adhered firmly, with the expression of osteocalcin and alkaline phosphatase, the cells gradually differentiate into mature osteoblasts, followed by continuously woven bone formation. In the process of bone remodeling, the gradual replacement of woven bone with lamellar bone depends on the interactions between osteoblasts and osteoclasts [12]. However, the differences between the natural wound-healing process and dental implant osseointegration lie in the topography, roughness, surface energy, chemical properties, and other elements of the implant surface that play an important role during this process $[13,14]$.

\subsection{Topography and Roughness of Implant Surface}

A great number of previous studies suggest that compared to the smooth surface, proper micron roughness serves to enhance osteoblast differentiation and increase bone-implant contact rate in vivo, even in the absence of osteogenic growth factors [15-18]. However, with the increase of surface roughness, the implant surface was also enlarged, which might aggravate bacterial colonization [19]. As a result, the balance between the required biological reaction and elimination of plaque accumulation around the implant needs to be considered [20]. In addition, roughness of micron surface topography to a certain extent could activate specific cell membrane receptors such as integrins, which serves as a communicator in the interactions between the extracellular matrix and the cytoskeleton [21, 22]. Previous studies indicate that surface roughness of natural bones is about $32 \mathrm{~nm}$, and pore size of epithelial basement membrane is about $70-100 \mathrm{~nm}$. Nanoscale roughened material 
might affect a variety of cells, such as epithelial cells, osteoblasts, fibroblasts, and so on [23]. Some studies show that the nanoscale surface materials are potent in altering the adhesion between the surface and the protein or cells. Moreover, Webster et al. found that nanosurface materials effectively improved the adhesion of vitronectin compared with the microscale surfaces. In addition, the surface of nanomaterials promotes osteoblast adhesion and proliferation [24, 25]. Also, Ellingsen et al. found that implants with nanosurface accelerate the bone formation around the implants [26].

\subsection{The Surface Wettability}

Wettability is another important feature of the material surface. This basic physical parameter can be quantified by the value of the surface contact angle (CA), which is first proposed by Thomas Young in 1805 [27]. Generally, a surface with a contact angle of less than $90^{\circ}$ is considered as a hydrophilic one and if the contact angle is equal to $0^{\circ}$, it is considered as a super-hydrophilic surface. On the contrary, the hydrophobic surface is defined as the one with the value of CA more than $90^{\circ}$.

The roughness would also influence the wettability of material surfaces. Previous studies have shown that the surface contact angle of the pure titanium was about $70-90^{\circ}$ regardless of surface roughness. However, after a serial surface roughening process including acid etching and sandblasting, the CA of the surface could reach up to $150^{\circ}$. Moreover, it is reported that the CA of the surface rarely exceeds $120^{\circ}$ if the material is only treated by chemical methods rather than roughening. Therefore, the hydrophobic surfaces with the CA ranging from 125 to $180^{\circ}$ often call for a combination of different treatments [28-30]. Surfaces with different wettability produce diverse biological effects of osseointegration, and the impact can be divided into the following four aspects: protein and biological macromolecular adhesion to the material surface, biological behavior of different cells on the surface, the formation of the bacteria biofilm, and in vivo study of osseointegration.

There are different views on the adhesion of protein on the surfaces with different wettability. Previous studies have shown that the fibronectin adhesion is much more facilitated on a hydrophilic surface than on the hydrophobic surface, and the fibronectin adhering to the hydrophilic surface can maintain a better biological activity which could promote osteoblast adhesion and differentiation [7, 31]. However, Tugulu et al. [32] found that the super-hydrophilic surface treated by diluted alkaline solution would reduce the adhesion of the fibrinogen and thereby reduce the inflammation around the implant, which enhances the potential of the promotion of implant osseointegration in vivo [33]. Besides, the adhesion of other proteins which play important roles in osteogenic differentiation such as vitronectin and type I collagen is also affected by surfaces with diverse characteristics [34].

More recently, some results demonstrate that the expression of genes related to the osteogenic differentiation of mesenchymal stem cells on the super-hydrophilic surface is higher than the level of the expression of the cells on the hydrophobic 
surface, indicating that the super-hydrophilic surface is more conducive to the osteogenic differentiation of mesenchymal stem cells than the hydrophobic surface $[17,35]$. It was also implied that super-hydrophilic surface can serve better to promote osteoblast maturation and mineralization [15, 36]. In 2005, Buser et al. [37] found that the super-hydrophilic surface modified SLA (modSLA) promoted the bone-implant contact rate in the early period ( 2 and 4 weeks) in vivo, while there was no difference between the experimental group and the control group 8 weeks later after the implantation. In 2007, Schwarz et al. [38] indicated that the formation of collagen fibers was observed on the super-hydrophilic surface modSLA of the implant in the first 4 days after the implantation. Two weeks later, bones formed around the super-hydrophilic surfaces became much denser than those formed on the control surfaces. Another experiment conducted on healthy adult volunteers showed that the implants with super-hydrophilic surface can promote bone integration after 2-4 weeks [39]. In addition, the adhesion and proliferation of the soft tissues around the implant such as epithelial cells and fibroblasts were simultaneously affected by the wettability of the surface. Similar results showed that superhydrophilic surface was helpful to form the rapid and compact soft tissue seal around the implant $[31,40]$. In summary, a large number of studies deliver a conclusion that the hydrophilic surface, especially the super-hydrophilic surface, exhibits preferable advantages in terms of promoting osseointegration in the early stage.

\subsection{The Common Methods of Surface Modifications}

Since the surface morphology and characteristics of the implant play important roles in the osseointegration process, researches on the surface of the implant are particularly indispensable. The surfaces with relatively similar features can be obtained even through several completely different processes, whereas the same process can fabricate two different types of surfaces only by changing their parameters [41]. Based on the topology and the average roughness value ( $\mathrm{Sa}$ ) of the material surface, Albrektsson and Wennerberg et al. [42] classified implant surfaces into four categories: smooth surface (Sa: 0-0.04 $\mu \mathrm{m})$, slightly rough surface (Sa: $0.5-1$ $\mu \mathrm{m})$, medium rough surface $(\mathrm{Sa}: 1-2 \mu \mathrm{m})$, and rough surface $(\mathrm{Sa}:>2 \mu \mathrm{m})$. Among them, the medium rough surface exhibited satisfactory clinical results in comparison with smooth surface and rough surface. In another way by some other researchers, the implant surfaces can be classified into three categories based on the surface roughness: large roughness surface (Sa greater than $10 \mu \mathrm{m}$ ), micro-sized roughness surface (Sa: 1-10 $\mu \mathrm{m}$ ), and nano-sized roughness surface [16]. Among them, the rough surface demonstrated satisfactory performance in the protein adhesion, the formation of extracellular matrix, the promotion of osteogenic differentiation and osseointegration in vivo, etc. Therefore, the roughening treatment is still prevalent in modifying the implant surface.

The following is the common practical roughening methods, such as plasma spraying, sand blasting, chemical etching, anodic oxidation, electrochemical 
micro-arc oxidation treatment method, and so on [41]. By virtue of plasma spraying technique, the osteoinductive hydroxyapatite (HA) particles with good biocompatibility and mechanical strength could adhere to the surface and roughen the surface at the same time. Moreover, it has been confirmed that osseointegration can be enhanced by this HA-coated surfaces [43].

Sandblasted and acid-etched (SLA) is a common roughening method employed in titanium dental implant surfaces, which forms the complex surface with considerate roughness and micro-roughness. The method mentioned above has been commercialized. Moreover, this approach has a "gold standard" in the industry: firstly, the surface of the implant is sandblasted by using aluminum oxide particles (250$500 \mu \mathrm{m}$ ), and then the specimen is etched by $\mathrm{HCl} / \mathrm{H}_{2} \mathrm{SO}_{4}$ solution of specific concentration. The average surface roughness (Ra) of the treated material is $1.5 \mu \mathrm{m}$ [44]. In 1990, Wilke et al. [45] first reported the in vivo application of SLA surface implants and found that the treatment could effectively improve the mechanical stability of the implant. Buser et al. [46] observed that the SLA surface treatment effectively increased the bone contact rate of the implant from small pigs in vivo compared to the titanium plasma spray method (titanium plasma spray, TPS). A body of evidence presented in published data indicates that pits with the diameter of 1-2 $\mu \mathrm{m}$ can be observed being distributed regularly on the SLA surface and that the processing method may raise the short-term bone contact rate of the implant after the implantation. In addition, a large number of studies have shown that the SLA surfaces of in vitro experiments, animal experiments, and clinical practices have achieved good results $[44,47]$. Besides, nanoscaled treatments on the implant surface not only affect the topography of the implant surface but also change the chemical properties of the material surface. There are several common nanotechnologies for surface processing, including physical compression method, self-assembled monolayers, chemical treatment method (acid, alkali, and hydrogen peroxide), nanoparticle deposition method, the anodic oxidation method, etc. [23].

There are several surface treatment methods which could enhance the wettability of the surface of the material. For example, Baier et al. [48] found that the use of radio-frequency glow discharge (RFGD) technology could effectively clean and disinfect the surface of inorganic materials, thus increasing the surface energy of the materials and strengthening the adhesion of the cells. It was reported that the superhydrophilic surface could be formed after an atmospheric plasma treatment on titanium foil. In this way, the contact angle could approach $0^{\circ}$, which greatly facilitates osteoblasts to spread on the treated surface [49]. But at the same time, some researchers believe that the chemical or plasma treatment yields satisfactory hydrophilicity that could not last for long in air [50].

However, by roughening and chemical treatment of the implant surface, the osteoconductivity could thus be enhanced. With the development of biochemical techniques, some specific short peptide fragments, proteins or growth factors, and other biologically active substance could be fixed onto the surface of the material so as to promote surface osteoinductivity. Currently used biologically active molecules 
currently fall into the following categories: cell adhesion molecules, such as those containing arginine-glycine-aspartic acid (RGD) peptide which could promote cell adhesion and extracellular matrix attachment; bone morphogenetic proteins (BMP) such as the application of BMP-2 and BMP-7, which have strong ability to promote bone formation; and growth factors, such as insulin-like growth factors (IGFs), transforming growth factor- $\beta$ (TGF- $\beta$ ), vascular endothelial growth factor (VEGF), fibroblast growth factor (FGF), and so on $[51,52]$.

\subsection{Polydopamine Surface Modification}

Marine mussels adhered to the surface of the metal, rock, and so many different materials even under humid conditions that rely on the main component of mussel adhesive protein (MAPs). The protein contains lysine and L-3,4dihydroxyphenylalanine (L-3,4-dihydroxyphenylalanine, L-DOPA). Inspired by the adhesion of mussels, a versatile poly dopamine (PDA) has been developed which could adhere to a wide range of organic and inorganic materials, such as metals, glasses, ceramics, and other synthetic polymers. In 2007, Lee et al. [53] found that by soaking the material in an alkaline $(\mathrm{pH}=8.5)$ dopamine solution, a layer of PDA membrane could be formed on the material surface, which also provided a platform for secondary material modification. The two-step method of covalent modification of the fixed type I collagen on the titanium surface by means of PDA coating promotes MC3T3-E1 cell adhesion and early osteogenic differentiation [54]. Lee et al. [55] used 15 short peptides derived from BMP-7 amino acid which is called bone formation peptide1 (BFP1) to modify the polylactic acid-glycolic acid (PLGA) copolymer surface through PDA coating. The modified materials were implanted to reconstruct the skull defects in mice. Eight weeks later, the modified materials exhibited enhanced new bone formation around the bone defects. In another animal experiment, human adipose-derived stem cells (hADSCs) cultured on the PDA and BMP-2-modified PLGA scaffold were implanted in mice skull defects, and the modified materials were also found to effectively enhance new bone formation [56]. It is reported that PDA coating is a biocompatible coating which can be reduced by PDA-modified PLLA of inflammation and immune responses [57].

\section{References}

1. Branemark R, Branemark P, Rydevik B, et al. Osseointegration in skeletal reconstruction and rehabilitation: a review. J Rehabil Res Dev. 2001;38:175.

2. Brắnemark P, Zarb G, Albrektesson T. Tissue-integrated prostheses. In: Quintessence Publishing Co., Inc., Chicago; 1985.

3. Brunette DM. Principles of cell behavior on titanium surfaces and their application to implanted devices[A]. In: Titanium in medicine. Berlin: Springer; 2001. p. 485-512. 
4. Sul Y-T. The significance of the surface properties of oxidized titanium to the bone response: special emphasis on potential biochemical bonding of oxidized titanium implant. Biomaterials. 2003;24:3893-907.

5. Elias CN, Oshida Y, Lima JH, et al. Relationship between surface properties (roughness, wettability and morphology) of titanium and dental implant removal torque. J Mech Behav Biomed Mater. 2008;1:234-42.

6. Ku SH, Park CB. Human endothelial cell growth on mussel-inspired nanofiber scaffold for vascular tissue engineering. Biomaterials. 2010;31:9431-7.

7. Wilson CJ, Clegg RE, Leavesley DI, et al. Mediation of biomaterial-cell interactions by adsorbed proteins: a review. Tissue Eng. 2005;11:1-18.

8. Marx RE. Platelet-rich plasma: evidence to support its use. J Oral Maxillofac Surg. 2004;62:489-96.

9. Terheyden H, Lang NP, Bierbaum S, et al. Osseointegration - communication of cells. Clin Oral Implants Res. 2012;23:1127-35.

10. Jansson E, Tengvall P. In vitro preparation and ellipsometric characterization of thin blood plasma clot films on silicon. Biomaterials. 2001;22:1803-8.

11. Witte MB, Barbul A. Role of nitric oxide in wound repair. Am J Surg. 2002;183:406-12.

12. Zelzer E, McLean W, Ng YS, et al. Skeletal defects in VEGF(120/120) mice reveal multiple roles for VEGF in skeletogenesis. Development. 2002;129:1893-904.

13. Colnot C, Romero DM, Huang S, et al. Molecular analysis of healing at a bone-implant interface. J Dent Res. 2007;86:862-7.

14. Ponche A, Bigerelle M, Anselme K. Relative influence of surface topography and surface chemistry on cell response to bone implant materials. Part 1: physico-chemical effects. Proc Inst Mech Eng H. 2010;224:1471-86.

15. Vlacic-Zischke J, Hamlet SM, Friis T, et al. The influence of surface microroughness and hydrophilicity of titanium on the up-regulation of TGFbeta/BMP signalling in osteoblasts. Biomaterials. 2011;32:665-71.

16. Le Guehennec L, Soueidan A, Layrolle P, et al. Surface treatments of titanium dental implants for rapid osseointegration. Dent Mater. 2007;23:844-54.

17. Olivares-Navarrete R, Hyzy SL, Hutton DL, et al. Direct and indirect effects of microstructured titanium substrates on the induction of mesenchymal stem cell differentiation towards the osteoblast lineage. Biomaterials. 2010;31:2728-35.

18. Cochran DL, Schenk RK, Lussi A, et al. Bone response to unloaded and loaded titanium implants with a sandblasted and acid-etched surface: a histometric study in the canine mandible. J Biomed Mater Res. 1998;40:1-11.

19. An YH, Friedman RJ. Concise review of mechanisms of bacterial adhesion to biomaterial surfaces. J Biomed Mater Res. 1998;43:338-48.

20. Albrektsson T, Wennerberg A. The impact of oral implants - past and future, 1966-2042. J Can Dent Assoc. 2005;71:327.

21. Zhao G, Raines AL, Wieland M, et al. Requirement for both micron- and submicron scale structure for synergistic responses of osteoblasts to substrate surface energy and topography. Biomaterials. 2007;28:2821-9.

22. Keselowsky BG, Wang L, Schwartz Z, et al. Integrin alpha(5) controls osteoblastic proliferation and differentiation responses to titanium substrates presenting different roughness characteristics in a roughness independent manner. J Biomed Mater Res A. 2007;80:700-10.

23. Mendonca G, Mendonca DB, Aragao FJ, et al. Advancing dental implant surface technology from micron- to nanotopography. Biomaterials. 2008;29:3822-35.

24. Webster TJ, Schadler LS, Siegel RW, et al. Mechanisms of enhanced osteoblast adhesion on nanophase alumina involve vitronectin. Tissue Eng. 2001;7:291-301.

25. Webster TJ, Ergun C, Doremus RH, et al. Specific proteins mediate enhanced osteoblast adhesion on nanophase ceramics. J Biomed Mater Res. 2000;51:475-83.

26. Ellingsen JE, Johansson CB, Wennerberg A, et al. Improved retention and bone-implant contact with fluoride-modified titanium implants. Int $\mathrm{J}$ Oral Maxillofac Implants. 2004;19:659-66. 
27. Young T. An essay on the cohesion of fluids. Phil Trans R Soc (London). 1805;95:65-87.

28. Rupp F, Scheideler L, Eichler M, et al. Wetting behavior of dental implants. Int J Oral Maxillofac Implants. 2011;26:1256-66.

29. Gittens RA, Olivares-Navarrete R, Cheng A, et al. The roles of titanium surface micro/nanotopography and wettability on the differential response of human osteoblast lineage cells. Acta Biomater. 2013;9:6268-77.

30. Marmur A. Hydro-hygro-oleo-omni-phobic? Terminology of wettability classification. Soft Matter. 2012;8:6867-70.

31. Gittens RA, Scheideler L, Rupp F, et al. A review on the wettability of dental implant surfaces II: biological and clinical aspects. Acta Biomater. 2014;10:2907-18.

32. Tugulu S, Lowe K, Scharnweber D, et al. Preparation of superhydrophilic microrough titanium implant surfaces by alkali treatment. J Mater Sci Mater Med. 2010;21:2751-63.

33. Tang L, Wu Y, Timmons RB. Fibrinogen adsorption and host tissue responses to plasma functionalized surfaces. J Biomed Mater Res. 1998;42:156-63.

34. Salasznyk RM, Williams WA, Boskey A, et al. Adhesion to vitronectin and collagen I promotes osteogenic differentiation of human mesenchymal stem cells. BioMed Res Int. 2004;2004:24-34.

35. Wall I, Donos N, Carlqvist K, et al. Modified titanium surfaces promote accelerated osteogenic differentiation of mesenchymal stromal cells in vitro. Bone. 2009;45:17-26.

36. Zhao G, Schwartz Z, Wieland M, et al. High surface energy enhances cell response to titanium substrate microstructure. J Biomed Mater Res A. 2005;74:49-58.

37. Buser D, Broggini N, Wieland M, et al. Enhanced bone apposition to a chemically modified SLA titanium surface. J Dent Res. 2004;83:529-33.

38. Schwarz F, Herten M, Sager M, et al. Histological and immunohistochemical analysis of initial and early osseous integration at chemically modified and conventional SLA titanium implants: preliminary results of a pilot study in dogs. Clin Oral Implants Res. 2007;18:481-8.

39. Lang NP, Salvi GE, Huynh-Ba G, et al. Early osseointegration to hydrophilic and hydrophobic implant surfaces in humans. Clin Oral Implants Res. 2011;22:349-56.

40. Scheideler L, Rupp F, Wendel HP, et al. Photocoupling of fibronectin to titanium surfaces influences keratinocyte adhesion, pellicle formation and thrombogenicity. Dent Mater. 2007;23:469-78.

41. Annunziata M, Guida L. The effect of titanium surface modifications on dental implant osseointegration. Front Oral Biol. 2015;17:62-77.

42. Albrektsson T, Wennerberg A. Oral implant surfaces: part 1 - review focusing on topographic and chemical properties of different surfaces and in vivo responses to them. Int J Prosthodont. 2004;17:536-43.

43. Darimont G, Cloots R, Heinen E, et al. In vivo behaviour of hydroxyapatite coatings on titanium implants: a quantitative study in the rabbit. Biomaterials. 2002;23:2569-75.

44. Szmukler-Moncler S, Perrin D, Ahossi V, et al. Biological properties of acid etched titanium implants: effect of sandblasting on bone anchorage. J Biomed Mater Res B Appl Biomater. 2004;68:149-59.

45. Wilke H, Claes L, Steinemann S. The influence of various titanium surfaces on the interface shear strength between implants and bone. Adv Biomater. 1990;9:309-14.

46. Buser D, Schenk R, Steinemann S, et al. Influence of surface characteristics on bone integration of titanium implants. A histomorphometric study in miniature pigs. J Biomed Mater Res. 1991;25:889-902.

47. Cochran DL, Buser D, Ten Bruggenkate CM, et al. The use of reduced healing times on ITI ${ }^{\circledR}$ implants with a sandblasted and acid-etched (SLA) surface. Clin Oral Implants Res. 2002;13:144-53.

48. Baier R, Meyer A, Natiella J, et al. Surface properties determine bioadhesive outcomes: methods and results. J Biomed Mater Res. 1984;18:337-55.

49. Duske K, Koban I, Kindel E, et al. Atmospheric plasma enhances wettability and cell spreading on dental implant metals. J Clin Periodontol. 2012;39:400-7. 
50. Rupp F, Axmann D, Ziegler C, et al. Adsorption/desorption phenomena on pure and Teflon® AF-coated titania surfaces studied by dynamic contact angle analysis. J Biomed Mater Res. 2002;62:567-78.

51. Kim T-I, Jang J-H, Kim H-W, et al. Biomimetic approach to dental implants. Curr Pharm Des. 2008;14:2201-11.

52. Avila G, Misch K, Galindo-Moreno P, et al. Implant surface treatment using biomimetic agents. Implant Dent. 2009;18:17-26.

53. Lee H, Dellatore SM, Miller WM, et al. Mussel-inspired surface chemistry for multifunctional coatings. Science. 2007;318:426-30.

54. Yu X, Walsh J, Wei M. Covalent immobilization of collagen on titanium through polydopamine coating to improve cellular performances of MC3T3-E1 cells. RSC Adv. 2013;4:7185-92.

55. Lee YJ, Lee JH, Cho HJ, et al. Electrospun fibers immobilized with bone forming peptide-1 derived from BMP7 for guided bone regeneration. Biomaterials. 2013;34:5059-69.

56. Ko E, Yang K, Shin J, et al. Polydopamine-assisted osteoinductive peptide immobilization of polymer scaffolds for enhanced bone regeneration by human adipose-derived stem cells. Biomacromolecules. 2013;14:3202-13.

57. Hong S, Kim KY, Wook HJ, et al. Attenuation of the in vivo toxicity of biomaterials by polydopamine surface modification. Nanomedicine. 2011;6:793-801.

Open Access This chapter is distributed under the terms of the Creative Commons Attribution 4.0 International License (http://creativecommons.org/licenses/by/4.0/), which permits use, duplication, adaptation, distribution and reproduction in any medium or format, as long as you give appropriate credit to the original author(s) and the source, provide a link to the Creative Commons license and indicate if changes were made.

The images or other third party material in this chapter are included in the work's Creative Commons license, unless indicated otherwise in the credit line; if such material is not included in the work's Creative Commons license and the respective action is not permitted by statutory regulation, users will need to obtain permission from the license holder to duplicate, adapt or reproduce the material. 